\title{
Non-Invasive Assessment of Coronary Microcirculation in Heart Transplantation
}

\author{
Laura De Michieli, Carlo Dal Lin, Francesco Tona* \\ Department of Cardiac, Thoracic, Vascular Sciences and Public Health, University of Padova, Padova, Italy \\ Email: *francesco.tona@unipd.it, *francescotona@hotmail.com
}

How to cite this paper: De Michieli, L., Dal Lin, C. and Tona, F. (2019) Non-Invasive Assessment of Coronary Microcirculation in Heart Transplantation. World Journal of Cardiovascular Diseases, 9, 796-811. https://doi.org/10.4236/wjcd.2019.911071

Received: August 12, 2019

Accepted: November 16, 2019

Published: November 19, 2019

Copyright ( 2019 by author(s) and Scientific Research Publishing Inc. This work is licensed under the Creative Commons Attribution International License (CC BY 4.0).

http://creativecommons.org/licenses/by/4.0/

Open Access

\begin{abstract}
Heart transplantation (HT) is an accepted treatment for end-stage heart failure (HF). Heart transplantation significantly increases survival, exercise capacity, quality of life and return to work in selected patients with advanced heart failure compared with conventional treatment. The survival rates have improved with the use of new immunosuppressive drugs, with a median survival after transplantation of approximately 11 years. The shortage of donor hearts represents a major limitation in this field. In addition many are the consequences of the limited effectiveness and complications of immunosuppressive therapy (i.e. antibody-mediated rejection, infection, hypertension, renal failure, malignancy and coronary artery vasculopathy). In particular, chronic rejection may occur months to years after the transplantation and is referred to as cardiac allograft vasculopathy (CAV). CAV occurs in $32 \%$ of the patients after 5 years and ensuing allograft failure from CAV eventually accounts for $30 \%$ of recipient deaths after transplantation. Cardiac allograft vasculopathy, involving coronary macro- and microcirculation, is caused by complicated interplay between immunologic and non-immunologic factors resulting in repetitive endothelial injury and localized sustained inflammatory response. Early diagnosis of microvascular dysfunction is substantial. In this review we analyze signs and symptoms of CAV presentation and the different methodologies to achieve an early and precise diagnosis. We will discuss invasive and non-invasive diagnostic tools and their specific role in evaluating graft's function, morphology, the presence of coronary artery disease and possible microcirculation involvement.
\end{abstract}

\section{Keywords}

Heart Transplantation, Coronary Microcirculation, Imaging, Rejection, Echocardiography 


\section{Introduction}

Heart transplantation (HT) is an accepted treatment for end-stage heart failure (HF). Although controlled trials have never been conducted, there is a consensus that transplantation significantly increases survival, exercise capacity, quality of life and return to work in selected patients with advanced heart failure compared with conventional treatment [1]. The survival rates have improved with the use of new immunosuppressive drugs, with a median survival after transplantation of approximately 11 years [2].

Heart transplantation is to be considered in end-stage HF patients with severe symptoms, a poor prognosis, and no remaining alternative treatment options. Patients must be motivated, well informed, emotionally stable and capable of complying with the intensive treatment required postoperatively. Many are the relative and absolute contraindications that must be analyzed case by case [3]. The shortage of donor hearts represents a major limitation in this field; in addition to this, the main challenges in transplantation are the consequences of the limited effectiveness and complications of immunosuppressive therapy (i.e. antibody-mediated rejection, infection, hypertension, renal failure, malignancy and coronary artery vasculopathy) [1].

Pre-transplant evaluation consists of several clinical, instrumental and laboratory evaluations; immune-compatibility testing should include $\mathrm{ABO}$ blood group typing. Although donor hearts are not selected on the basis of human leukocyte antigens (HLAs), screening for humoral sensitization is accomplished by means of panel-reactive antibody (PRA) testing to determine the presence of circulating anti-HLA antibodies [4]. Sensitization, although usually caused by pregnancy, blood transfusion, prior transplantation or placement of a ventricular assist device (VAD), occasionally occurs without an obvious sensitizing event, representing cross-reactivity between bacterial or viral epitopes and HLA antigens [4]. There are studies supporting the association of elevations in circulating antibodies (PRA > 10\%) with an increase in mortality, rejection, and the development of cardiac allograft vasculopathy (CAV) in the post-transplant period, as well as longer waiting times and increased in-list risk of mortality [3].

After heart transplantation, patients may manifest unique clinical complications (associated with the immunosuppressive therapy and cardiac allograft rejection) as well as atypical clinical presentations for infections and systemic inflammatory response syndrome.

Early diagnosis and appropriate intervention for allograft-related and non-allograft-related syndromes with significant morbidity and mortality are the keys to long-term survival of patients after transplantation [2].

Graft rejection can be classified according to its acuity in hyperacute rejection, acute rejection and chronic rejection. It can be as well classified according to the mechanism of the rejection: cell-mediated rejection or antibody-mediated rejection. [2]

Hyperacute rejection is mediated by preexisting antibodies to allogenic anti- 
gens and occurs within minutes to hours after the transplantation [5] while acute rejection can be categorized into cell-mediated and humoral-mediated rejection and occurs in the first week to several years after the transplantation. The inflammatory response of cell-mediated rejection consists mainly of T-cell lymphocytes [6] while humoral-mediated rejection consists of antibodies directed against the donor HLA [7].

Chronic rejection may occur months to years after the transplantation and can cause an irreversible graft dysfunction. In heart transplantation, chronic rejection is referred to as cardiac allograft vasculopathy (CAV); based on the ISHLT registry, CAV occurs in 32\% of the patients after 5 years and ensuing allograft failure from CAV eventually accounts for $30 \%$ of recipient deaths after transplantation [2]. The occurrence of CAV has not decreased despite advancements in immunosuppressive therapies and better prevention of acute rejection [8]. Moreover, according to international registry data, more than $50 \%$ of long-term mortality due to graft failure is not attributed to CAV; a fraction of those deaths could be related to underestimated CAV, underlining the need for improving CAV diagnosis long-term after transplant [9].

Generally speaking, chronic rejection manifests similar pathological findings in different organs: obliterative vasculopathy, infiltration of leukocytes, luminal occlusion, and a marked fibrotic response [10].

Specifically, cardiac allograft vasculopathy is caused by complicated interplay between immunologic and non-immunologic factors resulting in repetitive endothelial injury and localized sustained inflammatory response (Figure 1) [11].

$\mathrm{CAV}$ results from both antigen-dependent and antigen-independent immune

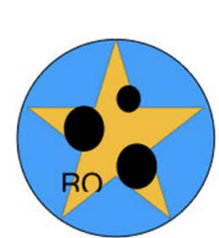

Oxidative Stress
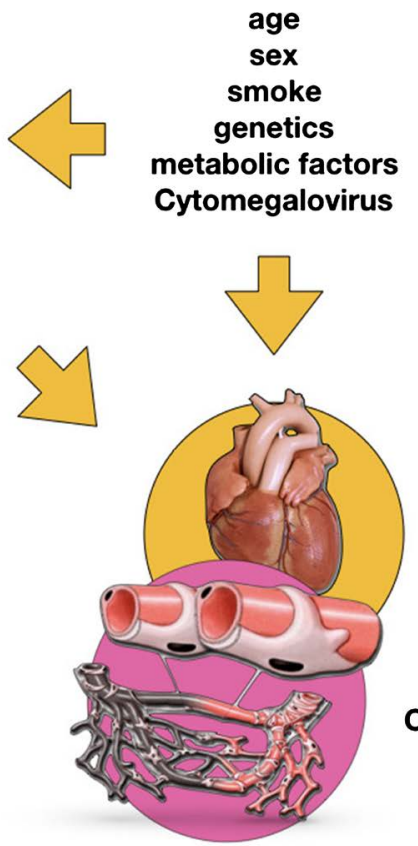

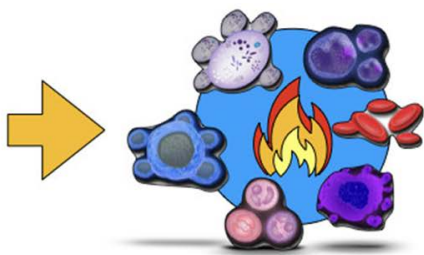

Inflammation

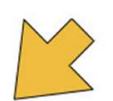

Coronary Microcirculation

CAV

Figure 1. The development of CAV is a multifactorial and complex process initiated by heterogeneous factors that ultimately cause inflammation, oxidative stress and endothelial injury, the precursor to CAV and coronary microvascular dysfunction ([11]-[43]). 
factors, and from autoimmune factors as well. Although numerous nonimmune entities are also implicated in the development of CAV, immune factors are the most important causes, given that CAV occurs within the arteries of the donor but not the recipient [12]. Nonimmunologic factors include cause of donor brain death, cytomegalovirus (CMV) infection, age, sex, obesity, dyslipidemia, hyperhomocysteinemia (HHcy), diabetes mellitus, hypertension, smoking and ischemia-reperfusion injury [2] [13].

In 2004, Caforio et al. found that risk factors for CAV onset were older donor, male donor, high rejection scores (RS) for severe grades and high cyclosporine at 3 months after transplantation. Risk factors for CAV severity and diffusion were higher donor weight, high prednisone dosage at 1 year and coronary disease pre-HT. High RS was an independent predictor for CAV onset, not severity/diffusion. This suggested an immune basis for CAV onset and nonimmune modulation for progression. High RS for severe grades were supposed to provide a predictor for patients at risk.

More recently, risk factors for development of CAV were proved to include also ischemic cardiomyopathy prior to transplant and re-transplant [14], while statins and mTOR inhibitors resulted preventative [15].

$\mathrm{CAV}$ is indeed a progressive and worsening condition characterized by diffuse, concentric thickening of the epicardial and intramyocardial coronary arteries; the obstructive process can progress to near-complete occlusion of the coronary arteries causing micro- and macroinfarctions [16]. The remodeling process can affect the epicardial coronary arteries primarily together with or without the intramyocardial arteries involvement, or the intramyocardial coronary microvasculature as primary and sole involvement. Predominant allograft microvascular dysfunction is detectable in around 15\% of patients after HT [16]. Early graft vascular lesions seem to interest mostly small coronary arteries, supporting the hypothesis that microvasculopathy is an immune-mediated phenomenon, similar to epicardial CAV, but which could precede the onset of epicardial CAV [17].

Histologically, repetitive endothelial injury and a localized sustained inflammatory response are followed by intimal hyperplasia and proliferation of vascular smooth muscle cells. Intramyocardial microvasculature shows not only concentric intimal thickening but the presence of plump endothelial cells [18]. Morphologic evaluation of microcirculation can be performed on endomyocardial biopsies to quantify the microvessels remodeling; the main indices utilized for the quantification of microvasculopathy are the microvascular density and arterioles or small arteries percent stenosis [16].

Early diagnosis of microvascular dysfunction in heart transplantation patients is substantial; as a matter of fact, in 2015 Tona et al. proved that microvascular dysfunction is independently associated with the onset of epicardial CAV, and associated with a higher risk of death, regardless of CAV onset [19].

So, how to perform an accurate and prompt diagnosis of microvascular and 
epicardial CAV?

Cardiac denervation at the time of heart transplantation usually prevents patients from experiencing angina, which is an important warning sign for heart disease. Because of this lack of typical symptoms, transplant patients with CAV usually present with silent myocardial infarction, loss of allograft function or sudden death [13]. Symptoms can be atypical, such as exertional dyspnea, gastrointestinal symptoms, or may have a severe initial presentation such as heart failure or even fatal arrhythmias [8] (Table 1).

In order to provide early diagnosis of chronic rejection, post-transplant patients undergo screening with coronary angiography starting at the first year post transplant and annually or bi-annually thereafter. Current guidelines indicate angiography, coupled with the assessment of graft function, as the imaging procedure of choice for CAV diagnosis and classification and to predict long-term prognosis.

\section{Evaluation of Graft's Function and Morphology as Indirect Signs of CAV}

Echocardiography is the first line imaging modality to assess graft function and morphology; in the immediate post-operative period, echocardiography enables identification of surgical complications and early allograft dysfunction, while in long-term follow-up, serial echocardiographic studies are useful to detect acute graft rejection, CAV and to monitor pulmonary artery systolic pressure [20].

Echocardiography can highlight new wall motion abnormalities that could be associated with the presence of CAV; late reduction of left ventricle ejection fraction (LVEF) is however often associated with progression of CAV with a subsequent poor prognosis [21].

Classical diastolic parameters can be altered in HT patients even without a

Table 1. Signs and symptoms of CAV ([13]-[45]).

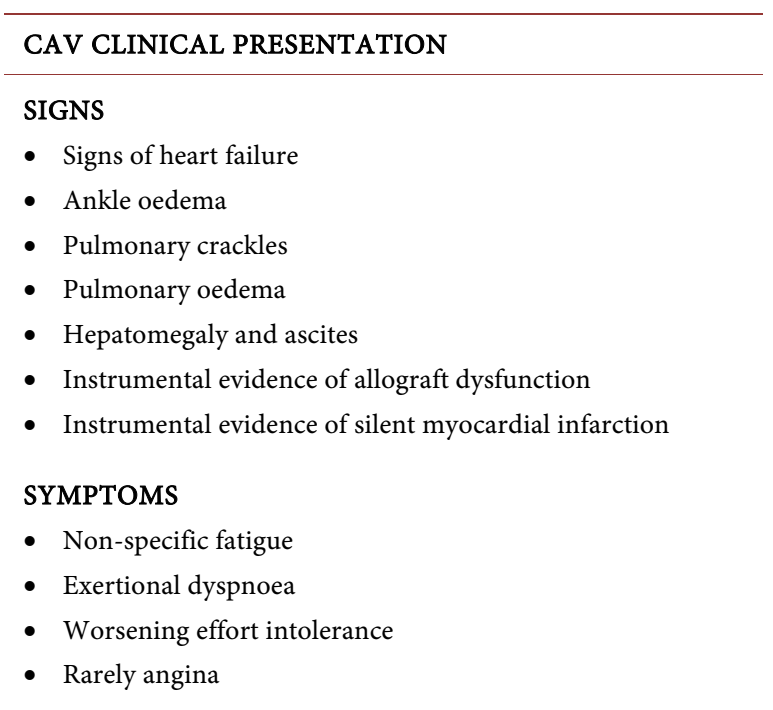


concomitant diastolic dysfunction; as a matter of facts, Tona et al. evaluated the role of myocardial performance index (MPI, meaning the sum of isovolumetric contraction time and isovolumetric relaxation time divided by ejection time) as a marker of long-term allograft dysfunction in 154 patients; they found a progressive increase in MPI during long-term follow-up in HT patients with preserved LV systolic function and MPI resulted higher in patients with multiple rejection episodes but no correlation was found with the occurrence of CAV [22].

So, the presence of alterations in LV systolic and diastolic function has an important role in the assessment of prognosis in HT patients, but it is not an accurate marker of graft rejection or CAV.

Worsening of longitudinal strain has been associated with acute cellular rejection [23]. Moreover, segmental longitudinal strain was found to be reduced in LV segments which showed inducible wall motion abnormalities during stress test and strain values could predict CAV [24].

Global longitudinal strain can be considered as a suitable parameter to diagnose subclinical allograft dysfunction, regardless of etiology, by comparing the changes occurring during serial evaluations [20].

Stress echocardiography has been reported to increase the specificity in detecting CAV. Dobutamine has been the most frequently used pharmacological stressor and a sensitivity between $70 \%$ and $80 \%$ to detect significant CAV at coronary angiography has been reported [20].

Three-dimensional echocardiography (3DE) could have an important role in assessing HT patients since it has been reported to be more accurate and reproducible than two-dimensional echocardiography in quantitating heart chambers volumes and mass. Moreover, during stress echocardiography, 3DE may improve the assessment of regional wall motion, possibly improving the accuracy of acute GR and CAV screening [20].

Cardiovascular magnetic resonance (CMR) imaging provides accurate and reproducible assessment of cardiac structure and function, independent of image plane and BMI, and in addition allows characterization of myocardial tissue [25]. CMR allows evaluation of kinetics alterations, left ventricle and right ventricle mass, volumes, coronary imaging and the in vivo tissue characterization; it also allows identification of areas of delayed hyperenhancement on post-contrast sequences identifying silent myocardial infarction in transplant recipients and distinguishing areas of fibrosis not-related to CAD [26]. In fact, LGE detects 'silent' infarcts in up to approximately a quarter of patients who have only mild vasculopathy angiographically; the majority of infarcts are found in mid and apical segments and is distributed across coronary territories [25].

\section{Evaluation of Epicardial Coronary Lesions and Microvascular Dysfunction}

In relation to coronary anatomy and physiology, as previously mentioned, angiography is the first invasive tool to be considered; the Stanford classification 
system is used to describe the morphology of coronary lesions from a discrete atherosclerosis to concentric arterial obliteration [20]. However, typical CAV features (such as its histological features and microvascular impairment) reduce the diagnostic sensitivity of coronary angiography [27]; coronary angiography can indeed underdiagnose the prevalence and extent of CAV due to the vascular remodeling which in an early stage does not necessarily reduce the luminal diameter [20].

Intravascular ultrasound (IVUS) has emerged as the gold standard for early detection of CAV thanks to high-resolution images of the cross-section of the vessel [20]. It allows the accurate quantitative assessment of lumen size, intimal thickening, vessel wall morphology, and composition [28] and therefore allows detection of angiographically silent early CAV. Combined imaging analysis of progression of angiographic lesions and IVUS-detected maximal intimal thickness (MIT), with a cutoff value of $\geq 0.35 \mathrm{~mm}$, between 1 and 5 years post-HT allows discriminating patients at high, intermediate, and low risk for adverse long-term cardiovascular outcomes [9]. IVUS should also be performed when there is discrepancy between non-invasive imaging tests and coronary angiography concerning the presence of CAV [20].

Intracoronary imaging with optical coherence tomography (OCT) adds additional spatial resolution that may provide further diagnostic benefit, although it is a currently a research rather than a clinical tool [29].

Coronary flow reserve (CFR), meaning the ratio between resting and maximal possible flow in coronary arteries, is an important functional parameter commonly used to investigate the pathophysiology of coronary circulation. CFR is dependent on the combined effects of epicardial coronary flow and coronary microvascular function. Therefore, impaired CFR may reflect the presence of coronary microvascular dysfunction in the absence of obstructive coronary artery narrowing.

The evaluation of endothelial function and CFR have been investigated invasively by intracoronary Doppler flow wire (IDFW) in many studies, but it is not suitable to detect early onset of endothelial alterations or to be repeated many times during follow-up; it may provide a valuable functional assessment of the microvasculature in CAV, but it is an invasive and expensive procedure [16].

In 2006, Tona et al. demonstrated for the first time that CFR evaluated by contrast-enhanced transthoracic echocardiography (CE-TTE) in the left descendent coronary artery (LAD) was a feasible and accurate noninvasive tool for CAV detection [30]; this method was previously shown and validated against Doppler flow wire measurements in coronary artery disease [31]. CFR by CE-TTE has indeed been shown to correlate with angiographically detectable coronary artery lesion severity as well as intracoronary Doppler flow wire measurements [31], and to stratify the risk of cardiac events in HT patients [32].

Moreover, CFR assessment by CE-TTE could detect CAV defined as MIT $\geq$ $0.5 \mathrm{~mm}$. Microvascular dysfunction, as assessed by CFR, was indeed proved to 
be correlated with intimal hyperplasia measured by IVUS in patients with physiologically normal epicardial coronary arteries, suggesting the possible concordant involvement of both macro- and microvascular system in early CAV [33].

In 2015, Tona et al. demonstrated that coronary microvascular dysfunction assessed after the first year post-HT was likely to become a future independent predictor of new onset epicardial CAV [19]. CFR evaluated by transthoracic Doppler echocardiography (TDE) provided indeed prognostic information on clinically stable HT recipients: a CFR $\leq 2.5$ was independently associated with a higher probability of new onset CAV and a higher probability of death, regardless of CAV onset [19].

Non-invasive detection of impaired CFR in HT recipients has been studied also with cardiac magnetic resonance; this examination though is not available for all transplantation recipients due to frequent renal impairment that limits the utilization of gadolinium contrast-based agents; moreover, the high cardiac rate, due to denervation of the heart, may limit the quality of images. In general population with suspected coronary artery disease, the clinical role of stress CMR has been validated in a multicenter study (MR-IMPACT [34]) in 241 patients demonstrating that perfusion CMR is either equivalent or superior to perfusion single photon emission computed tomography (SPECT) for the detection of coronary artery lesions.

In transplant recipients without demonstrable CAV on IVUS, resting myocardial blood flow (MBF) evaluated by CMR is elevated and, consequently, transplant recipients have decreased myocardial perfusion reserve (MPR). In one study, an MPR of $>2.3$ post-transplant was found to exclude angiographic CAV [35] [36]. In these patients, stress perfusion CMR with adenosine was tested in different studies, demonstrating reduced myocardial blood flow in patients with CAV compared with the normal population, allowing stratification of vascular disease severity. The application of stress-CMR to assess reduced CFR in transplant patients has been used and described by Muehling and co-workers [35]; they proved that non-invasive determination of myocardial perfusion reserve with magnetic resonance perfusion imaging (MRPI) allows exclusion of transplant arteriopathy and closely correlates with the invasive data on coronary flow reserve. Furthermore, they could identify patients with allograft vasculopathy using only the Endo/Epi ratio when LV hypertrophy and/or prior rejection were excluded. In addition, they thought that MRPI in combination with magnetic resonance cine imaging for cardiac function might be a good method for routine surveillance of patients after cardiac transplantation.

On the basis of the previously reported correlation between Doppler velocities and CMR phase contrast measurement, Kennedy et al. compared in 17 transplants recipients the CFR, measured by ratio between rest flow in the coronary sinus and during hyperemia (obtained by dipyridamole infusion), with angiography findings [37]. In this study, during hyperemia, a significant difference was seen between the control group and the "severe disease" CAV group. 
In 2009, in a large population study [38], besides the quantification of myocardial perfusion reserve, the estimation in HT patients of the mean diastolic strain rate using Strain-Encoding MR resulted a useful parameter for the detection of chronic allograft vasculopathy. In combination with the clinical evaluation, perfusion reserve and diastolic strain represented early markers of this disease and therefore they were considered as an effective tool for the routine surveillance of HTx-recipients, reducing the number of patients in need of invasive testing.

In 2014, Miller CA et al. [39] studied 48 transplant recipients with invasive fractional flow reserve (FFR), microvascular resistance and IVUS to assess performance of CMR first-pass perfusion to detect CAV. They proved that, in a comprehensive assessment of cardiac structure and function in the medium to long term after transplantation, CMR-based MPR was independently predictive of both epicardial and microvascular components of CAV. Furthermore, the diagnostic performance of CMR-MPR was significantly higher than that of coronary angiography, the current clinical screening technique.

Computer tomographic (CT) coronary angiography can be employed to exclude relevant CAV. This imaging modality offers the possibility of evaluating the coronary lumen, as well as the wall thickness and intimal hyperplasia, with a potential for early CAV detection. [20] CT coronary calcium score may have utility in the evaluation of post-transplant coronary calcification that is associated with the presence of CAV as defined by current ISHLT guidelines, yet the absence of coronary calcification does not exclude CAV [29]. Dynamic myocardial perfusion imaging during pharmacological vasodilation allows quantification of myocardial perfusion and may provide a non-invasive alternative to Positron Emission Tomography (PET) imaging for the detection of microvascular disease in the future [20].

About the field of nuclear cardiac imaging, in 2003 Yen-Wen Wu et al. [40] studied 47 patients at a mean of 34 months after transplantation who received dobutamine thallium-201 $\left({ }^{201} \mathrm{TI}\right)$ singol photon emission computed tomography (SPECT), echocardiography and coronary angiography within one months of each other. Dobutamin ${ }^{201}$ TI SPECT was found to be a useful method for detecting patients with significant CAV and assessing prognosis; the sensitivity, specificity, positive predictive value and negative predictive value of SPECT for the detection of significant angiographic CAV were $89 \%, 71 \%, 42 \%$ and $96 \%$, respectively [40]. Large reversible perfusion defect was a significant predictor of cardiac death $(\mathrm{p}=0.002)$ [40].

On the other hand, Thompson et al. [41] more recently showed that adenosine stress/rest technetium-99m tetrofosmin-gated SPECT was not a sensitive test for detection of CAV in heart transplant recipients; diastolic dysfunction, assessed by SPECT, was not shown to be associated with development of CAV. As a predominantly qualitative technique, perfusion SPECT does not detect CAV until flow-limiting disease of epicardial coronary arteries is present [29]. 
Cardiac position emission tomography, on the other hand, has been used extensively to study graft perfusion after cardiac transplantation. As mentioned before for CMR perfusion evaluation, normal graft perfusion differs from native heart perfusion, and is increased at rest by approximately $40 \%$; resting MBF decreases however with time after transplant, and may eventually return near normal levels [29].

Flow quantification is suited to evaluate CAV because it can determine global $\mathrm{MBF}$, which detects balanced or diffuse epicardial and microvascular coronary disease. Cardiac PET is the clinical gold standard for non-invasive quantification of MBF and myocardial flow reserve (MFR). It has also been proved the prognostic value of reduced stress MBF and MFR on PET to predict adverse events after heart transplantation [42].

In 2018, Chih et al. evaluated forty patients that underwent coronary angiography, rubidium 82 (Rb-82) PET, multivessel intravascular ultrasound (IVUS), and intracoronary hemodynamics. CAV was defined as International Society of Heart and Lung Transplantation CAV1-3 on angiography and maximal intimal thickness $\geq 0.5 \mathrm{~mm}$ on IVUS. They found a correlation between noninvasive PET myocardial flow and invasive coronary flow measures in heart transplant patients; PET demonstrated high diagnostic performance for detecting epicardial intimal disease in CAV. Optimal PET diagnostic cutoffs for CAV were rate-pressure product-adjusted myocardial flow reserve (cMFR) < 2.9 , stress $\mathrm{MBF}<2.3$, and coronary vascular resistance $>55$; they showed high sensitivity for IVUS-determined CAV of combined PET assessment for any one abnormal PET cMFR, stress MBF, or CVR parameter, as well as high specificity for any two abnormal parameters. These results supported a highly promising role for Rb-82 PET in noninvasive assessment of CAV [43].

They proposed a diagnostic algorithm with cardiac PET as a discriminant for CAV unlikely, CAV possible or CAV likely and subsequent indication to consider or perform coronary angiography and IVUS.

Comparable results were found in the same year by Bravo et al. who evaluated 94 HT recipients (prognostic cohort), including 66 who underwent invasive coronary angiography and PET within 1 year (diagnostic cohort). They demonstrated that multiparametric cardiac PET evaluation including quantification of MBF provides improved detection and gradation of CAV severity over standard myocardial perfusion assessment and is predictive of major adverse events [44].

\section{Conclusion}

In conclusion, cardiac allograft vasculopathy, including epicardial arteries disease and coronary microcirculation impairment, remains to date one of the main challenges in long term management of HT patients causing reduction of their life expectancy. Proper early diagnosis is substantial, in order to provide closer follow-up and therapeutic changes. Epicardial coronary arteries involvement is just of the possible manifestation of CAV; microvascular dysfunction is indeed 
an early and prognosis-correlated aspect of the disease. As discussed previously, non-invasive assessment of coronary microcirculation with different methods has been proved to be important in CAV diagnosis and prognosis evaluation, in order to provide close and accurate follow-up in HT recipients.

\section{Conflicts of Interest}

The authors declare no conflicts of interest regarding the publication of this paper.

\section{References}

[1] Ponikowski, P., Voors, A.A., Anker, S.D., Bueno, H., Cleland, J.G.F., Coats, A.J.S., Falk, V., González-Juanatey, J.R., Harjola, V.-P., Jankowska, E.A., Jessup, M., Linde, C., Nihoyannopoulos, P., Parissis, J.T., Pieske, B., et al. (2016) ESC Guidelines for the Diagnosis and Treatment of Acute and Chronic Heart Failure: The Task Force for the Diagnosis and Treatment of Acute and Chronic Heart Failure of the European Society of Cardiology (ESC) Developed with the Special Contribution of the Heart Failure Association (HFA) of the ESC. European Heart Journal, 37, 2129-2200. https://doi.org/10.1093/eurheartj/ehw128

[2] Birati, E.Y. and Rame, J.E. (2014) Post-Heart Transplant Complications. Critical Care Clinics, 30, 629-637. https://doi.org/10.1016/j.ccc.2014.03.005

[3] Mehra, M.R., Canter, C.E., Hannan, M.M., Semigran, M.J., Uber, P.A., Baran, D.A., Danziger-Isakov, L., Kirklin, J.K., Kirk, R., Kushwaha, S.S., Lund, L.H., Potena, L., Ross, H.J., Taylor, D.O., Verschuuren, E.A.M., et al. (2016) The 2016 International Society for Heart Lung Transplantation Listing Criteria for Heart Transplantation: A 10-Year Update. The Journal of Heart and Lung Transplantation, 35, 1-23. https://doi.org/10.1016/j.healun.2015.10.023

[4] Mehra, M., Kobashigawa, J., Starling, R., Russell, S., Uber, P., Parameshwar, J., Mohacsi, P., Augustine, S., Aaronson, K. and Barr, M. (2006) Listing Criteria for Heart Transplantation: International Society for Heart and Lung Transplantation Guidelines for the Care of Cardiac Transplant Candidates-2006. The Journal of Heart and Lung Transplantation, 25, 1024-1042.

https://doi.org/10.1016/j.healun.2006.06.008

[5] Iyer, A., Kumarasinghe, G., Hicks, M., Watson, A., Gao, L., Doyle, A., Keogh, A., Kotlyar, E., Hayward, C., Dhital, K., Granger, E., Jansz, P., Pye, R., Spratt, P. and Macdonald, P.S. (2011) Primary Graft Failure after Heart Transplantation. Journal of Transplantation, 2011, Article ID: 175768. https://doi.org/10.1155/2011/175768

[6] Ingulli, E. (2010) Mechanism of Cellular Rejection in Transplantation. Pediatric Nephrology, 25, 61-74. https://doi.org/10.1007/s00467-008-1020-X

[7] Colvin, M.M., Cook, J.L., Chang, P., Francis, G., Hsu, D.T., Kiernan, M.S., Kobashigawa, J.A., Lindenfeld, J., Masri, S.C., Miller, D., O’Connell, J., Rodriguez, E.R., Rosengard, B., Self, S., White-Williams, C., et al. (2015) Antibody-Mediated Rejection in Cardiac Transplantation: Emerging Knowledge in Diagnosis and Management: A Scientific Statement From the American Heart Association. Circulation, 131, 1608-1639. https://doi.org/10.1161/CIR.0000000000000093

[8] McCartney, S.L., Patel, C. and Del Rio, J.M. (2017) Long-Term Outcomes and Management of the Heart Transplant Recipient. Best Practice \& Research Clinical Anaesthesiology, 31, 237-248. https://doi.org/10.1016/j.bpa.2017.06.003

[9] Potena, L., Masetti, M., Sabatino, M., Bacchi-Reggiani, M.L., Pece, V., Prestinenzi, 
P., Dall'Ara, G., Taglieri, N., Saia, F., Fallani, F., Magnani, G., Rapezzi, C. and Grigioni, F. (2015) Interplay of Coronary Angiography and Intravascular Ultrasound in Predicting Long-Term Outcomes after Heart Transplantation. The Journal of Heart and Lung Transplantation, 34, 1146-1153. https://doi.org/10.1016/j.healun.2015.01.990

[10] Weiss, M.J. (2008) Mechanisms of Chronic Rejection in Cardiothoracic Transplantation. Frontiers in Bioscience, 13, 2980. https://doi.org/10.2741/2903

[11] Caforio, A.L.P., Tona, F., Fortina, A.B., Angelini, A., Piaserico, S., Gambino, A., Feltrin, G., Ramondo, A., Valente, M., Iliceto, S., Thiene, G. and Gerosa, G. (2004) Immune and Nonimmune Predictors of Cardiac Allograft Vasculopathy Onset and Severity: Multivariate Risk Factor Analysis and Role of Immunosuppression. American Journal of Transplantation, 4, 962-970.

https://doi.org/10.1111/j.1600-6143.2004.00434.x

[12] Costello, J.P. and Mohanakumar, T. (2013) Mechanisms of Chronic Cardiac Allograft Rejection. Texas Heart Institute Journal, 40, 5.

[13] Ramzy, D., Rao, V., Brahm, J., Miriuka, S., Delgado, D. and Ross, H.J. (2005) Cardiac Allograft Vasculopathy: A Review. Canadian Journal of Surgery, 48, 319-327.

[14] Lund, L.H., Edwards, L.B., Dipchand, A.I., Goldfarb, S., Kucheryavaya, A.Y., Levvey, B.J., Meiser, B., Rossano, J.W., Yusen, R.D., Stehlik, J. and International Society for Heart and Lung Transplantation (2016) The Registry of the International Society for Heart and Lung Transplantation: Thirty-Third Adult Heart Transplantation Report-2016; Focus Theme: Primary Diagnostic Indications for Transplant. The Journal of Heart and Lung Transplantation: The Official Publication of the International Society for Heart Transplantation, 35, 1158-1169. https://doi.org/10.1016/j.healun.2016.08.017

[15] Skorić, B., Čikeš, M., Ljubas Maček, J., Baričević, Ž., Škorak, I., Gašparović, H., Biočina, B. and Miličić, D. (2014) Cardiac Allograft Vasculopathy: Diagnosis, Therapy, and Prognosis. Croatian Medical Journal, 55, 562-576. https://doi.org/10.3325/cmj.2014.55.562

[16] Tona, F., Perazzolo Marra, M., Fedrigo, M., Famoso, G., Bellu, R., Thiene, G., Gerosa, G., Angelini, A. and Iliceto, S. (2012) Recent Developments on Coronary Microvasculopathy after Heart Transplantation: A New Target in the Therapy of Cardiac Allograft Vasculopathy. Current Vascular Pharmacology, 10, 206-215. https://doi.org/10.2174/157016112799304987

[17] Hiemann, N.E., Wellnhofer, E., Knosalla, C., Lehmkuhl, H.B., Stein, J., Hetzer, R. and Meyer, R. (2007) Prognostic Impact of Microvasculopathy on Survival after Heart Transplantation: Evidence from 9713 Endomyocardial Biopsies. Circulation, 116, 1274-1282. https://doi.org/10.1161/CIRCULATIONAHA.106.647149

[18] Clausell, N., Butany, J., Molossi, S., Lonn, E., Gladstone, P., Rabinovitch, M. and Daly, P.A. (1995) Abnormalities in Intramyocardial Arteries Detected in Cardiac Transplant Biopsy Specimens and Lack of Correlation with Abnormal Intracoronary Ultrasound or Endothelial Dysfunction in Large Epicardial Coronary Arteries. Journal of the American College of Cardiology, 26, 110-119. https://doi.org/10.1016/0735-1097(95)00134-L

[19] Tona, F., Osto, E., Famoso, G., Previato, M., Fedrigo, M., Vecchiati, A., Perazzolo Marra, M., Tellatin, S., Bellu, R., Tarantini, G., Feltrin, G., Angelini, A., Thiene, G., Gerosa, G. and Iliceto, S. (2015) Coronary Microvascular Dysfunction Correlates with the New Onset of Cardiac Allograft Vasculopathy in Heart Transplant Patients with Normal Coronary Angiography: Microvascular Dysfunction Correlates with CAV. American Journal of Transplantation, 15, 1400-1406. 
https://doi.org/10.1111/ajt.13108

[20] Badano, L.P., Miglioranza, M.H., Edvardsen, T., Colafranceschi, A.S., Muraru, D., Bacal, F., Nieman, K., Zoppellaro, G., Marcondes Braga, F.G., Binder, T., Habib, G., Lancellotti, P., Sicari, R., Cosyns, B., et al. (2015) European Association of Cardiovascular Imaging/Cardiovascular Imaging Department of the Brazilian Society of Cardiology Recommendations for the Use of Cardiac Imaging to Assess and Follow Patients after Heart Transplantation. European Heart Journal: Cardiovascular Imaging, 16, 919-948. https://doi.org/10.1093/ehjci/jev139

[21] Wilhelmi, M., Pethig, K., Wilhelmi, M., Nguyen, H., Strüber, M. and Haverich, A. (2002) Heart Transplantation: Echocardiographic Assessment of Morphology and Function after More than 10 Years of Follow-Up. The Annals of Thoracic Surgery, 74, 1075-1079. https://doi.org/10.1016/S0003-4975(02)03833-X

[22] Tona, F., Caforio, A.L.P., Piaserico, S., Bontorin, M., Simone, G.D., Leone, M.G., Fortina, A.B., Gambino, A., Feltrin, G., Calzolari, D., Angelini, A., Peserico, A., Thiene, G., Gerosa, G. and Iliceto, S. (2005) Abnormal Total Ejection Isovolume Index as Early Noninvasive Marker of Chronic Rejection in Heart Transplantation. Transplant International, 18, 303-308. https://doi.org/10.1111/j.1432-2277.2004.00051.x

[23] Podrouzkova, H., Bedanova, H., Tretina, M., Korinek, J., Stepanova, R., Hruskova, J., Nemec, P., Konecny, T. and Orban, M. (2015) Decrease in Longitudinal Strain in Heart Transplant Recipients Is Associated with Rejection. Biomedical Papers of the Medical Faculty of the University Palacky, Olomouc, Czechoslovakia, 159, 601-606. https://doi.org/10.5507/bp.2015.020

[24] Marciniak, A., Eroglu, E., Marciniak, M., Sirbu, C., Herbots, L., Droogne, W., Claus, P., Dhooge, J., Bijnens, B. and Vanhaecke, J. (2007) The Potential Clinical Role of Ultrasonic Strain and Strain Rate Imaging in Diagnosing Acute Rejection after Heart Transplantation. European Journal of Echocardiography, 8, 213-221. https://doi.org/10.1016/j.euje.2006.03.014

[25] Reid, A.B., Waldron, N., Schmitt, M. and Miller, C.A. (2015) The Value of Cardiovascular Magnetic Resonance in Heart Transplant Patients. Current Cardiology Reports, 17, 58. https://doi.org/10.1007/s11886-015-0612-X

[26] Steen, H., Merten, C., Refle, S., Klingenberg, R., Dengler, T., Giannitsis, E. and Katus, H.A. (2008) Prevalence of Different Gadolinium Enhancement Patterns in Patients after Heart Transplantation. Journal of the American College of Cardiology, 52, 1160-1167. https://doi.org/10.1016/j.jacc.2008.05.059

[27] Johnson, D.E., Alderman, E.L., Schroeder, J.S., Gao, S.-Z., Hunt, S., DeCampli, W.M., Stinson, E. and Billingham, M. (1991) Transplant Coronary Artery Disease: Histopathologic Correlations with Angiographic Morphology. Journal of the American College of Cardiology, 17, 449-457.

https://doi.org/10.1016/S0735-1097(10)80114-7

[28] Tsutsui, H., Ziada, K.M., Schoenhagen, P., Iyisoy, A., Magyar, W.A., Crowe, T.D., Klingensmith, J.D., Vince, D.G., Rincon, G., Hobbs, R.E., Yamagishi, M., Nissen, S.E. and Tuzcu, E.M. (2001) Lumen Loss in Transplant Coronary Artery Disease Is a Biphasic Process Involving Early Intimal Thickening and Late Constrictive Remodeling: Results From a 5-Year Serial Intravascular Ultrasound Study. Circulation, 104, 653-657. https://doi.org/10.1161/hc3101.093867

[29] McDiarmid, A.K., Plein, S. and Ross, H.J. (2016) Emerging Imaging Techniques after Cardiac Transplantation. The Journal of Heart and Lung Transplantation, 35, 1399-1411. https://doi.org/10.1016/j.healun.2016.06.017 
[30] Tona, F., Caforio, A.L.P., Montisci, R., Angelini, A., Ruscazio, M., Gambino, A., Ramondo, A., Thiene, G., Gerosa, G. and Iliceto, S. (2006) Coronary Flow Reserve by Contrast-Enhanced Echocardiography: A New Noninvasive Diagnostic Tool for Cardiac Allograft Vasculopathy: Coronary Flow Reserve in Heart Transplantation. American Journal of Transplantation, 6, 998-1003. https://doi.org/10.1111/j.1600-6143.2006.01298.x

[31] Caiati, C., Montaldo, C., Zedda, N., Montisci, R., Ruscazio, M., Lai, G., Cadeddu, M., Meloni, L. and Iliceto, S. (1999) Validation of a New Noninvasive Method (Contrast-Enhanced Transthoracic Second Harmonic Echo Doppler) for the Evaluation of Coronary Flow Reserve. Journal of the American College of Cardiology, 34, 1193-1200. https://doi.org/10.1016/S0735-1097(99)00342-3

[32] Tona, F. (2006) Coronary Flow Velocity Pattern and Coronary Flow Reserve by Contrast-Enhanced Transthoracic Echocardiography Predict Long-Term Outcome in Heart Transplantation. Circulation, 114, I-49-I-55. https://doi.org/10.1161/CIRCULATIONAHA.105.001321

[33] Tona, F., Osto, E., Tarantini, G., Gambino, A., Cavallin, F., Feltrin, G., Montisci, R., Caforio, A.L.P., Gerosa, G. and Iliceto, S. (2010) Coronary Flow Reserve by Transthoracic Echocardiography Predicts Epicardial Intimal Thickening in Cardiac Allograft Vasculopathy: Coronary Flow Reserve in Heart Transplantation. American Journal of Transplantation, 10, 1668-1676. https://doi.org/10.1111/j.1600-6143.2010.03160.x

[34] Schwitter, J., Wacker, C.M., van Rossum, A.C., Lombardi, M., Al-Saadi, N., Ahlstrom, H., Dill, T., Larsson, H.B.W., Flamm, S.D., Marquardt, M. and Johansson, L. (2008) MR-IMPACT: Comparison of Perfusion-Cardiac Magnetic Resonance with Single-Photon Emission Computed Tomography for the Detection of Coronary Artery Disease in a Multicentre, Multivendor, Randomized Trial. European Heart Journal, 29, 480-489. https://doi.org/10.1093/eurheartj/ehm617

[35] Muehling, O.M., Wilke, N.M., Panse, P., Jerosch-Herold, M., Wilson, B.V., Wilson, R.F. and Miller, L.W. (2003) Reduced Myocardial Perfusion Reserve and Transmural Perfusiongradient in Heart Transplant Arteriopathyassessed by Magnetic Resonance Imaging. Journal of the American College of Cardiology, 42, 1054-1060. https://doi.org/10.1016/S0735-1097(03)00924-0

[36] Muehling, O.M., Panse, P., Jerosch-Herold, M., Wilson, B.V., Wilson, R.F., Wilke, N.M. and Miller, L.W. (2005) Cardiac Magnetic Resonance Perfusion Imaging Identifies Transplant Arteriopathy by a Reduced Endomyocardial Resting Perfusion. The Journal of Heart and Lung Transplantation: The Official Publication of the International Society for Heart Transplantation, 24, 1122-1123. https://doi.org/10.1016/j.healun.2004.08.022

[37] Kennedy, K., Dick, A., Drangova, M., Raval, A., Mahoney, C., Karlik, S. and Pflugfelder, P.W. (2007) Magnetic Resonance Measurements of Coronary Flow Reserve in Heart Transplant Recipients: An Exploratory Study of the Relationship to Coronary Angiographic Findings. Journal of Cardiovascular Magnetic Resonance, 9, 701-707. https://doi.org/10.1080/10976640701198980

[38] Korosoglou, G., Osman, N.F., Dengler, T.J., Riedle, N., Steen, H., Lehrke, S., Giannitsis, E. and Katus, H.A. (2009) Strain-Encoded Cardiac Magnetic Resonance for the Evaluation of Chronic Allograft Vasculopathy in Transplant Recipients. American Journal of Transplantation, 9, 2587-2596. https://doi.org/10.1111/j.1600-6143.2009.02769.x

[39] Miller, C.A., Sarma, J., Naish, J.H., Yonan, N., Williams, S.G., Shaw, S.M., Clark, D., Pearce, K., Stout, M., Potluri, R., Borg, A., Coutts, G., Chowdhary, S., 
McCann, G.P., Parker, G.J.M., et al. (2014) Multiparametric Cardiovascular Magnetic Resonance Assessment of Cardiac Allograft Vasculopathy. Journal of the American College of Cardiology, 63, 799-808. https://doi.org/10.1016/j.jacc.2013.07.119

[40] Wu, Y.-W., Yen, R.-F., Lee, C.-M., Ho, Y.-L., Chou, N.-K., Wang, S.-S. and Huang, P.-J. (2005) Diagnostic and Prognostic Value of Dobutamine Thallium-201 Single-Photon Emission Computed Tomography after Heart Transplantation. The Journal of Heart and Lung Transplantation, 24, 544-550.

https://doi.org/10.1016/j.healun.2003.12.004

[41] Thompson, D., Koster, M.J., Wagner, R.H., Heroux, A. and Barron, J.T. (2012) Single Photon Emission Computed Tomography Myocardial Perfusion Imaging to Detect Cardiac Allograft Vasculopathy. European Heart Journal: Cardiovascular Imaging, 13, 271-275. https://doi.org/10.1093/ejechocard/jer270

[42] Mc Ardle, B.A., Davies, R.A., Chen, L., Small, G.R., Ruddy, T.D., Dwivedi, G., Yam, Y., Haddad, H., Mielniczuk, L.M., Stadnick, E., Hessian, R., Guo, A., Beanlands, R.S., deKemp, R.A. and Chow, B.J.W. (2014) Prognostic Value of Rubidium-82 Positron Emission Tomography in Patients After Heart Transplant. Circulation: Cardiovascular Imaging, 7, 930-937. https://doi.org/10.1161/CIRCIMAGING.114.002184

[43] Chih, S., Chong, A.Y., Erthal, F., deKemp, R.A., Davies, R.A., Stadnick, E., So, D.Y., Overgaard, C., Wells, G., Mielniczuk, L.M. and Beanlands, R.S.B. (2018) PET Assessment of Epicardial Intimal Disease and Microvascular Dysfunction in Cardiac Allograft Vasculopathy. Journal of the American College of Cardiology, 71, 1444-1456. https://doi.org/10.1016/j.jacc.2018.01.062

[44] Bravo, P.E., Bergmark, B.A., Vita, T., Taqueti, V.R., Gupta, A., Seidelmann, S., Christensen, T.E., Osborne, M.T., Shah, N.R., Ghosh, N., Hainer, J., Bibbo, C.F., Harrington, M., Costantino, F., Mehra, M.R., et al. (2018) Diagnostic and Prognostic Value of Myocardial Blood Flow Quantification as Non-Invasive Indicator of Cardiac Allograft Vasculopathy. European Heart Journal, 39, 316-323. https://doi.org/10.1093/eurheartj/ehx683

[45] Ambrosi, P., Kreitmann, B., Riberi, A., Lambert, M., Pankert, M. and Habib, G. (2016) Chronic Heart Failure in Heart Transplant Recipients: Presenting Features and Outcome. Archives of Cardiovascular Diseases, 109, 254-259.

https://doi.org/10.1016/j.acvd.2016.01.003 


\section{Abbreviations}

3DE Three-dimensional echocardiography

CAV Cardiac Allograft Vasculopathy

CE-TTE Contrast-Enhanced Transthoracic Echocardiography

CFR Coronary Flow Reserve

CMR Cardiac Magnetic Resonance

CT Computer tomographic

FFR fractional flow reserve

GR Graft Rejection

HF Heart Failure

HLAs Human Leukocyte Antigens

HT Heart Transplantation

IDFW Intracoronary Doppler Flow Wire

IVUS Intravascular Ultrasound

LGE Late Gadolinium Enhancement

LV Left Ventricle

LVEF Left Ventricular Ejection Fraction

MBF myocardial blood flow

MIT Maximal Intimal Thickness

MPR myocardial perfusion reserve

MRPI magnetic resonance perfusion imaging

OCT Optical Coherence Tomography

PET Positron Emission Tomography

PRA Panel-Reactive antibody

RS Rejection Scores

SPECT single photon emission computed tomography

VAD Ventricular Assist Device 